\title{
RÔLE ET RESPONSABILITÉS DES HAUTS DIRIGEANTS FACE AUX CHANGEMENTS CLIMATIQUES : RÉFLEXIONS À PARTIR DU CAS DE BP
}

\author{
Corinne Gendron, Bernard Girard, Silvester Ivanaj, Vera Ivanaj et Alice Friser
}

ESKA | « Entreprises et histoire 》

2017/1 nº 86 | pages 34 à 53

ISSN 1161-2770

ISBN 9782747227056

Article disponible en ligne à l'adresse :

https://www.cairn.inforevue-entreprises-et-histoire-2017-1-page-34.htm

Distribution électronique Cairn.info pour ESKA.

(C) ESKA. Tous droits réservés pour tous pays.

La reproduction ou représentation de cet article, notamment par photocopie, n'est autorisée que dans les limites des conditions générales d'utilisation du site ou, le cas échéant, des conditions générales de la licence souscrite par votre établissement. Toute autre reproduction ou représentation, en tout ou partie, sous quelque forme et de quelque manière que ce soit, est interdite sauf accord préalable et écrit de l'éditeur, en dehors des cas prévus par la législation en vigueur en France. Il est précisé que son stockage dans une base de données est également interdit. 


\section{RÔLE ET RESPONSABILITÉS DES HAUTS DIRIGEANTS FACE AUX CHANGEMENTS CLIMATIQUES : RÉFLEXIONS À PARTIR DU CAS DE BP}

\section{par Corinne GENDRON}

UQAM et ICN

Bernard GIRARD

UQAM

Silvester IVANAJ

CEREFIGE ICN Business School Université de Lorraine

Vera IVANAJ

CEREFIGE ENSIC Université de Lorraine

et Alice FRISER

UQAM

En s'imposant comme un enjeu majeur du XXle siècle, le changement climatique en appelle à l'engagement et à la responsabilité des entreprises. Cependant comment imaginer une effectivité de cet engagement si les échelons les plus élevés de l'entreprise ne s'engagent pas à leur tour et n'intègrent pas dans la stratégie même de l'entreprise les questions de climat? À partir d'une analyse du cas des dirigeants de BP, suite à la tragédie de 2010 au large du Mexique, les auteurs révèlent les verrous que l'intrication des rôles et devoirs de la gouvernance pose à toute stratégie climatique sérieuse dans les grandes entreprises.

\section{INTRODUCTION}

Le dernier rapport du Groupe d'experts intergouvernemental sur l'évolution du cli- mat (GIEC) est catégorique : les émissions de $\mathrm{CO}_{2}$ imputables à l'usage des combustibles fossiles et aux procédés industriels contribuent à près de $80 \%$ à l'accroissement total mondial de gaz à effet de serre 
(GES). Par conséquent, si les réserves de combustibles fossiles disponibles sont entièrement utilisées ${ }^{1}$, la température moyenne mondiale augmentera de 4 à $5^{\circ} \mathrm{C}$ d'ici $2100^{2}$. Or c'est une hausse mondiale maximale de $2^{\circ} \mathrm{C}$ qu'il ne faut absolument pas dépasser pour éviter des conséquences auxquelles il serait difficile de s'adapter.

Heureusement, en décembre 2015, après plus de vingt ans de discussions, c'est cet objectif - une hausse maximale de $2^{\circ} \mathrm{C}$ d'ici 2100 - que les 195 États présents lors de la $21^{\mathrm{e}}$ conférence des parties (CPO21) se sont donné avec l'accord de Paris. Cet engagement arrive à point puisque poursuivre notre consommation actuelle à l'échelle mondiale pendant trente ans suffirait à dépasser la limite des $2^{\circ} \mathrm{C}^{3}$. Au cours des dernières années, nombre d'entreprises, incluant les plus grandes compagnies gazières et pétrolières, ont également affirmé leur engagement en la matière, faisant de la lutte contre le réchauffement climatique la principale préoccupation environnementale de la planète ${ }^{4}$.

Mais des questions se posent : comment ces compagnies comptent-elles s'y prendre pour réduire leurs émissions de $\mathrm{CO}_{2}$ en sachant qu'avec l'accroissement de la population mondiale et surtout avec l'amélioration de son niveau de vie, on estime que la demande énergétique augmentera d'au moins $50 \%$ et que la demande en électricité doublera d'ici 2030 ? comment ces compagnies envisagent-elles les solutions préconisées par les experts pour lutter contre le réchauffement climatique, telles que la décarbonation de la production d'électricité par la capture et le stockage de carbone ou encore les énergies renouvelables et alternatives? et plus fondamentalement encore, alors que l'on assiste à un essor fulgurant des combustibles fossiles non conventionnels, quel crédit accordent-elles réellement à la lutte contre le réchauffement climatique?

C'est que depuis quelques années, l'engagement environnemental de ces compagnies semble pour le moins ambigu. Tel est le cas de la compagnie British Petroleum (BP) qui, suite à la catastrophe survenue sur sa plate-forme pétrolière Deepwater Horizon en avril 2010, a vu son image d'entreprise modèle voler en éclats. Le décalage entre la tragédie, sa gestion, les déclarations de sa haute direction d'une part et l'image publique de l'entreprise d'autre part fut pour la population un brusque réveil, sinon vécue comme une trahison.

C'est ce qui a inspiré la démarche de cet article qui vise à comprendre comment les dirigeants d'entreprise envisagent leurs responsabilités vis-à-vis des changements climatiques. Pour ce faire, on s'attachera d'abord à rappeler le déroulement de la tragédie de Deepwater Horizon et la façon dont elle a été gérée. Puis, en mobilisant les analyses qui ont été faites de la gouvernance de BP lors de la tragédie, on mettra en perspective l'image exemplaire de $\mathrm{BP}$ en matière de protection de l'environnement et de sécurité. Ensuite, sur la base de ses 21

\footnotetext{
${ }^{1}$ Incluant les combustibles fossiles dits non conventionnels, tels que les gaz de schiste ou le pétrole issu de sables bitumineux dont l'exploitation génère encore davantage de GES. Voir note 2.

${ }^{2}$ Sous la direction de l'équipe de rédaction principale, R. K. Pachauri et L. A. Meyer, « Changements climatiques 2014 : Rapport de synthèse », in Contribution des Groupes de travail I, II et III au cinquième Rapport d'évaluation du Groupe d'experts intergouvernemental sur l'évolution du climat, Genève, GIEC, 2014. En ligne : <http://www. ipcc.ch/pdf/assessment-report/ar5/syr/SYR_AR5_FINAL_full_fr.pdf>

${ }^{3}$ Selon Jean Jouzel, climatologue membre du GIEC, cité dans S. Huet, « Lutte contre le réchauffement : le GIEC pas à sec », Libération, 2 novembre 2014. En ligne : <http://www.liberation.fr/sciences/2014/11/02/lutte-contre-lerechauffement-le-giec-pas-a-sec_1134766>

${ }^{4} \mathrm{~S}$. Bertrand, « Les grands projets énergétiques et la communication », in V. Lehmann et B. Motulsky (dir.), Communication et grands projets : les nouveaux défis, Québec, Éditions PUQ, 2013, p. 198-209.
} 
discours officiels prononcés avant la tragédie, on cherchera à voir comment Tony Hayward, le dirigeant de BP de 2007 à 2010 , envisageait les changements climatiques et sa responsabilité pour y faire face. Ce développement à trois volets nous permettra enfin de dégager une réflexion plus large sur la responsabilité sociétale du dirigeant d'entreprise.

\section{LA TRAGÉDIE DE DEEPWATER HORIZON}

Le 20 avril 2010, dans le golfe du Mexique, explosait la plate-forme pétrolière Deepwater Horizon. En plus de tuer 11 ouvriers et d'en blesser plusieurs autres, l'accident provoqua un incendie ainsi qu'une marée noire sans précédent. La fuite ne fut stoppée que plusieurs mois plus tard, le 19 septembre, après que pas moins de 4,9 millions de barils de pétrole se sont déversés dans l'océan. Considérée comme la pire marée noire de l'histoire américaine, la fuite a provoqué des conséquences environnementales, sociales et économiques considérables. La faune et la flore ont été profondément perturbées, tandis que plusieurs réserves naturelles furent endommagées. L'utilisation de dispersants chimiques bioaccumulables a aussi suscité des critiques ${ }^{5}$. Ainsi, comme l'expliquent Carolyn Windsor et Patty McNicholas :

"While the GOM oil spill has had an unprecedented impact on the human health, environment and wild life of the region, perhaps the most insidious impact was 'the cure being, in many ways, worse that the disease' ${ }^{6}$. In an attempt to deal with the approximately 5 million barrels of oil and 500000 tons of gas released into the Gulf, BP used more than 1,8 million gallons of toxic chemical dispersants, which were mixed into the water and sprayed from the air to disperse the oil. Dispersants, however, do not remove the oil they simply disperse it throughout the water".

Les populations locales perdirent leurs principales sources de revenus (pêche et tourisme). Quant à BP, elle subit une chute importante de son cours boursier, simultanément à la baisse de sa cote de crédit ${ }^{7}$. Elle allait aussi devoir faire face à des amendes et à des demandes d'indemnisation en plus des coûts de remise en état de l'environnement.

Au lendemain de la catastrophe du golfe du Mexique, les autorités américaines ont créé une commission d'enquête, la National Commission on the BP Deepwater Horizon Oil Spill and Offshore Drilling, devant laquelle les dirigeants du groupe pétrolier ont témoigné et se sont exprimés ${ }^{8}$. Le rapport de cette commission donne ainsi à voir le discours et l'idéologie des dirigeants des grandes entreprises multinationales à travers les personnalités différenciées qui se sont succédé à la tête d'une entreprise dont l'image publique a soudainement basculé.

Tout autant que l'explosion et l'ampleur de la marée noire, la gestion de la crise par la direction de BP dévoila une image de l'entreprise aux antipodes de celle qu'elle s'était forgée avec son slogan «Beyond Petroleum : au-delà du pétrole ». Tout d'abord, BP n'a eu de cesse de minimiser la

\footnotetext{
${ }^{5}$ C. Windsor et P. McNicholas, "The BP Gulf Oil Spill: Public and Corporate Governance Failures”, 11th A-CSEAR Conference, University of Wollongong, 2012, p. 35.

${ }^{6}$ A. Juhasz, Black Tide: The Devastating Impact of the Gulf Oil Spill, Hoboken, New Jersey, John Wiley \& Sons, Inc., 2011, p. 89.

${ }^{7}$ Ibid.

${ }^{8}$ Le rapport de la Deepwater Commission (2011) se trouve en ligne : https://www.gpo.gov/fdsys/pkg/GPO-OILCOMMISSION/pdf/GPO-OILCOMMISSION.pdf. Voir aussi G. W. Conk, "Diving into the Wreck: BP and Kenneth Feinberg's Gulf Coast Gambit”, Roger Williams University Law Review, vol. 17, n 137, 2012.
} 
tragédie : ses estimations publiques de la fuite ne dépassaient pas 1000 à 5000 barils par jour, alors qu'un document interne rendu public en juin 2010 estimait plutôt la fuite à 100000 barils par jour. BP a aussi minimisé les difficultés à régler le problème: la première solution proposée, la construction d'un dôme, a dû être abandonnée alors qu'aucune solution de rechange n'était prévue ${ }^{9}$. La fuite s'est donc poursuivie pendant de longs mois, au point de mettre l'administration Obama dans l'embarras ${ }^{10}$. Enfin, BP a été critiquée pour son manque de transparence, que la publication sur son site web officiel de photos retouchées de son centre de crise n'a fait qu'aggraver ${ }^{11}$.

Au milieu de cette tempête, les déclarations du directeur général Tony Hayward ont jeté de l'huile sur le feu ${ }^{12}$. Le 3 mai, il affirme sur la chaîne de télévision américaine CBS : «Ce n'est pas notre accident, mais il est de notre responsabilité de nous en occuper, d'arrêter la fuite, de nous occuper du pétrole en surface, de nous assurer qu'il n'y ait pas de dégâts environnementaux, ou qu'ils soient minimes $»^{13}$. Le 14 mai, dans un entretien au quotidien The Guardian, le dirigeant estime l'ampleur de la catastrophe en ces termes : « Le golfe du Mexique est un très grand océan. La quantité de pétrole et de dispersant que nous y mettons est minuscule par rapport au volume d'eau total ». Estimation réitérée quelques jours plus tard, le 18 mai, alors qu'il explique sur les ondes de la chaîne britannique Sky News : «Je pense que l'impact environnemental de ce désastre sera probablement très, très modeste. C'est impossible de le dire pour le moment, et nous effectuerons, dans le cadre de l'après-catastrophe, une évaluation environnementale très détaillée. Mais tout ce que nous constatons pour le moment suggère que l'impact environnemental d'ensemble sera très, très modeste $»$. Enfin, le 30 mai, alors qu'il cherche à s'excuser pour le désastre, il affirme sur la chaine américaine $\mathrm{NBC}$, en faisant référence aux ouvriers et aux populations touchées par le désastre : « Nous sommes désolés pour le bouleversement que cela a provoqué dans leurs vies. Personne ne veut plus que moi que cela se termine. J'aimerais retrouver ma vie d'avant ». La controverse suscitée par ces déclarations culmine alors qu'en pleine tourmente, Tony Hayward prend des vacances en Angleterre en participant à une régate autour de l'Île de Wight le 19 juin.

La tragédie de Deepwater Horizon a frappé non seulement par son ampleur, mais aussi par le fait qu'elle mettait en cause une entreprise qui avait construit son image de marque sur l'excellence de ses performances environnementales ${ }^{14}$. Comme l'expliquent Miriam A. Cherry et Judd Sneirson :

"BP had a dismal safety record, with many accidents that could have been

\footnotetext{
${ }^{9}$ F. Lüdeke-Freund et D. Zvezdov, “The Manager's Job at BP: Decision Making and Responsibilities on the High Seas", International Journal of Case Studies in Management, vol. 11, n 3, 2013, p. 3.

${ }^{10}$ M. A. Cherry et J. Sneirson, "Beyond Profit: Rethinking Corporate Social Responsibilty and Greenwashing after the BP Oil Disaster", Tulane Law Review, vol. 85, n 4, 2011, p. 983-1083.

${ }^{11}$ Pour se donner une image plus réactive face à la crise, des photomontages ont été réalisés à partir de photographies du centre de gestion de crise de BP prises dans des circonstances «normales » avant l'incident, et des photos d'hélicoptères, de tour de contrôle et d'employés photographiés en pleine action. Lorsque le public s'est rendu compte que ces éléments avaient été ajoutés sur les photographies, celles-ci ont été retirées du site web de BP et des excuses ont alors été formulées. Pour en savoir davantage : http://www.telegraph.co.uk/news/earth/energy/ oil/7904221/BP-admits-it-Photoshopped-official-images-as-oil-spill-cut-and-paste-row-escalates.html.

12 Cette collection de citations est reprise de l'article « Tony Hayward, serial-gaffeur et piètre communicant », Libération, 26 juillet 2010 (source : AFP) : <http://www.liberation.fr/terre/0101648950-tony-hayward-serial-gaffeur-et-pietre-communicant> consulté le 11 juin 2013.

${ }^{13} \mathrm{Au}$ moment de l'accident, BP a rejeté la faute sur Transocean, qui rejeta la faute sur Cameron et Halliburton.

${ }^{14}$ M. A. Cherry et J. Sneirson, "Beyond Profit", art. cit., p. 992.
} 
avoided through better equipment maintenance and increased precautions. Ironically, the company at the same time mounted a highly successful advertising campaign, portraying itself as environmentally friendly, or at the very least not as bad as other oil companies, and a champion of environmental causes. The marketing worked perfectly: BP went from a staid middle-of-the pack oil company to one that consistently ranked in the top-ten most profitable multinational corporations in the world. It even ranked highly for its treatment of employees and became a darling of corporate social responsibility advocates" 15 .

\section{BP ET L'ENVIRONNEMENT : UN BILAN CONTROVERSÉ DERRIÈRE UNE CAMPAGNE PROMOTIONNELLE RÉUSSIE}

En 2000, BP s'engagea dans une imposante campagne de 200 millions de dollars visant à se positionner comme une entreprise chef de file en matière d'environnement et de sécurité à travers le slogan Beyond Petroleum. Elle fut la première entreprise pétrolière à reconnaître le lien entre l'utilisation d'énergie et les changements climatiques et à mettre autant l'accent sur le potentiel des énergies renouvelables. Elle soutint par ailleurs l'action de la Global Reporting Initiative ${ }^{16}$. La campagne fut un grand succès et, couplée à une série d'acquisitions, propulsa l'entreprise au rang des plus importantes entreprises mondiales; au cours du mandat de Lord Browne, le prix des actions de BP doubla, et l'agence à l'origine de la campagne fut submergée de demandes d'autres entreprises désireuses de refaçonner leur image ${ }^{17}$.

"Not only were most American consumers taken in, but so were legal academic commentators, environmentalists, and corporate social responsibility watch dogs. For example, the vice president and chief social investment strategist at Calvert, one of the largest socially responsible mutual funds in the country, noted in a media interview that she loved the environment and so would go out of her way to fill up her car at a BP gasoline station. [...] 'Beyond Petroleum' was for many years an unqualified success" $"$.

Toutefois, malgré son image verte, BP essuie en parallèle une série d'accidents environnementaux dont certains ont causé la mort d'ouvriers bien qu'ils soient demeurés peu connus ${ }^{19}$. En 2002, BP fut poursuivie pour avoir falsifié des rapports d'inspection d'une raffinerie de Los Angeles. En mars 2005, une explosion et un incendie ont emporté 15 ouvriers et blessé des centaines d'autres à la raffinerie de Texas City ${ }^{20}$. En 2006, deux fuites se produisirent en Alaska, dans la baie de Prudhoe, en raison d'une corrosion d'équipements non entretenus. Une explosion a aussi eu lieu sur une plateforme pétrolière en Azerbaïdjan en septembre 2008, et BP a pu évacuer ses 212 employés à temps. Mais elle a été critiquée

\footnotetext{
15 Ibid.

${ }^{16}$ C. Windsor et P. McNicholas, "The BP Gulf Oil Spill”, art. cit., p. 25.

${ }^{17}$ M. A. Cherry et J. Sneirson, "Beyond Profit", art. cit., p. 1006.

${ }^{18}$ Ibid., p. 1008.

${ }^{19}$ Le responsable de la raffinerie et du marketing, Iain Conn, admettait le 22 avril 2010 : "A bad track record leaves you with a long shadow, and we have to deal with that" (Guy Chazan, cité par M. A. Cherry et J. Sneirson, "Beyond Politics", art. cit., p. 995).

${ }^{20}$ M. A. Cherry et J. Sneirson, "Beyond Profit", art. cit., p. 996 indiquent : "An investigation after the accident found that the routine maintenance that was necessary - and which would have prevented the accident - had not been performed because it was deemed too expensive".
} 
pour avoir limité les informations auprès du public et de ses partenaires, tandis que son rapport annuel ne mentionnait même pas l'explosion ${ }^{21}$. Tous ces accidents engagèrent la responsabilité de $\mathrm{BP}$, démontrant des irrégularités dans la gestion de ses opérations et le non-respect de plusieurs législations environnementales et de santé et sécurité.

Plus troublant encore, plusieurs témoignages et rapports illustrent à quel point la culture de l'entreprise faisait peu de cas de la conformité, de l'environnement et de la sécurité face aux considérations budgétaires. Un rapport du panel chargé d'analyser l'accident de la raffinerie de Texas City, le BP US Refineries Independent Safety Review, reconnaît en 2007 que "a common theme [...] was a failure to follow BP's own procedures and an unwillingness to stop work when something was wrong" 22 . De même, "the report stated that while BP had 'aspirational goals' of no accidents and no harm, the company had not provided an effective leadership or a process of safety as a core value across its five refineries in the US. Further, BP had not effectively shown that it held senior executives, line managers and supervisors accountable for safety performance across its five US refineries. The report pointed out several other weaknesses with BP's safety performance, in contrast to the portrayal in its sustainability reports of a company genuinely committed to the safety of its workforce" 23 . En fait, c'est même une culture contraire à la sécurité qui prévalait chez BP :

"The internal investigation found that the company had a "pattern of [...] inti- midating workers who raised safety or environmental concerns. It said managers shaved maintenance costs by using aging equipment for as long as possible". The report also "quoted an employee who said employees felt forced to skip key diagnostics, including pressure testing, pipeline cleaning and corrosion checks". The same news story also detailed the tale of one whistleblower who was summarily fired for expressing safety concerns when he attempted to report hazardous conditions on the pipeline - conditions that could well have led to an explosion" 24 .

Cette culture est confirmée par une inspection en 2009 qui faisait suite à l'accident de Texas City. "A former employee, Ross MacFarlane, who was the health and safety manager on several BP rigs, claims that employees had 'high incentive to find shortcuts and take risks [...]. You only ever got questioned about why you couldn't spend less - never more""25. Bower rapporte pour sa part que la philosophie de Lord Browne, le prédécesseur de Tony Hayward, était « plus pour moins », de manière à réduire de façon systématique les coûts d'exploitation :

"Hundreds of engineers were sacked. Budgets for safety and maintenance were slashed. Skilled oil men resigned in disgust. [...] Doug Ford, an American responsible for BP's refineries, attacked what he believed was an increasingly badly-run organisation. After Lord Browne ignored his warnings about the consequences of cost-cutting, Mr. Ford resigned and others followed [...]. To

${ }^{21}$ C. Windsor et P. McNicholas, "The BP Gulf Oil Spill”, art. cit., rapportent ainsi : "In January 2009 BP blamed a 'bad cement job' for the gas leak, which bears a resemblance to Tony Hayward partly blaming a 'bad cement job' by contractor Halliburton for the GOM OCS incident". Voir aussi A. Juhasz, Black Tide, op. cit.

${ }^{22}$ G Chazan (2010), cité par M. A. Cherry et J. Sneirson, "Beyond Profit", art. cit., p. 1001.

${ }^{23}$ C. Windsor et P. McNicholas, "The BP Gulf Oil Spill”, art. cit., p. 33, en s'appuyant sur les analyses de J. Unerman et B. O'Dwyer, "The business case for regulation of corporate social responsibility and accountability", Accounting Forum, 31, 4, December 2007, p. 332-353.

${ }^{24}$ M. A. Cherry et J. Sneirson, "Beyond Profit", art. cit., p. 997.

${ }^{25}$ Ibid., p. 998. 
satisfy Lord Browne's 'more for less' mantra, Mr. Manzoni zealously pruned safety and maintenance costs" 26 .

Ainsi, devant les conclusions de ces rapports d'enquête quant aux manques et faiblesses des pratiques de $\mathrm{BP}$ en matière de conformité, de sécurité et de protection de l'environnement, et bien évidemment suite à l'incident dramatique du 20 avril 2010, plusieurs acteurs de la société civile soupçonneux à l'égard de la campagne Beyond Petroleum accusèrent ouvertement l'entreprise d'écoblanchiment (voir image 1). Tel est le cas en particulier de Greenpeace qui couronna les efforts de l'entreprise par le Emerald Paintbrush pour souligner le caractère superficiel de son engagement envers les énergies renouvelables notamment, alors que ses slogans publicitaires s'appuyaient principalement sur ces énergies : " from the earth to the sun, and everything in between », ou encore " the best way out of the energy fix is an energy mix ». Le bilan de Greenpeace mettait aussi en évidence le fait que, parmi les entreprises, BP était le plus important émetteur de $\mathrm{CO}_{2}$ au monde ${ }^{27}$.

De la même manière, des investisseurs responsables tels que Henderson Global Investors, Generation Investment Management ou Walden Asset Management se détournèrent de BP sur la base de leurs analyses internes en raison de l'orientation de l'entreprise ou encore compte tenu de sa piètre performance réelle en matière de sécurité et d'environnement ${ }^{28}$.
Image 1 : Le mix énergétique de BP ou une entreprise d'écoblanchiment (Greenpeace, 2008)

\section{Energy mix or PR fix?}

BP's actual investments in 2008

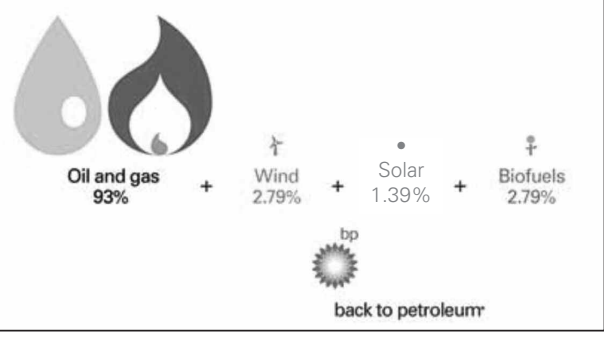

Néanmoins, malgré les insuffisances que les rapports d'enquête précités pointaient en matière d'environnement et de sécurité chez BP, Lord Browne, son dirigeant de 1995 à 2007, semblait engagé visà-vis de la question environnementale. Et bien que l'incident du 20 avril soit survenu alors qu'il était CEO de BP, Tony Hayward, son successeur, avait fait de la sécurité son cheval de bataille.

\section{LES DIRIGEANTS DE BP : QUEL RÔLE POUR QUELLE RESPONSABILITÉ ?}

Dans ses mémoires, Lord Browne présente son engagement environnemental comme une véritable croisade ${ }^{29}$. Même si le discours où il reconnaissait les changements climatiques qu'il prononça à Stanford fut

\footnotetext{
${ }^{26}$ Cité par C. Windsor et P. McNicholas, “The BP Gulf Oil Spill”, art. cit., p. 30.

${ }^{27}$ Greenpeace, "BP wins coveted 'Emerald Paintbrush' award for worst greenwash of 2008”, 22 décembre 2008, consulté le 4 novembre 2011 sur http://www.greenpeace.org.uk/blog/climate/bps-wins-coveted-emerald-paintbrush-award-worst-greenwash-2008-20081218. "The reality is that BP is one of the world's largest single corporate emitters. In 2007 alone the company released over 63 million tons of $\mathrm{CO}_{2}$ into the earth's atmosphere, roughly equivalent to the emissions of Portugal. But while their adverts announce the arrival of a fresh approach, BP boss Tony Hayward continues to describe alternative energy only as 'a valuable option for the future,".

${ }^{28}$ C. Windsor et P. McNicholas, "The BP Gulf Oil Spill”, art. cit., p. 35.

${ }^{29}$ Comme 1'expliquent M. A. Cherry et J. Sneirson, "Beyond Profit", art. cit., p. 1004 au sujet des mémoires de Lord Browne : "executives' stories show that the individual may have a significant impact on the way that a particular type of collective social organization - in this instance, a business - carries out its policies and goals".
} 
accueilli avec scepticisme et dérision, il était convaincu de la pertinence de ce positionnement, qui situait la firme en marge des autres acteurs de son secteur:

"I was the chief executive of an oil company and I was about to become an environmental activist. The message had to be clear and there had to be no doubt that BP was set on creating a new future" 30 .

$\mathrm{Au}$ vu des programmes écologiques, du soutien des groupes environnementaux et de la promotion de la diversité au sein de son entreprise, on a l'impression que la philosophie de Lord Browne est directement inspirée d'un manuel de responsabilité sociale ${ }^{31}$ :

"During all my time with BP, there was a debate on the wider purpose of business $[\ldots]$. Increasing short-term profits is often thought to be the same as creating 'shareholder value' [...]. But I began to understand that this way of thinking erodes a commitment to the longer term. After all, shareholder value is not about returns and growth rates alone; it is also about how long a company can keep growing [...]. A business must be useful to society and be seen as such. This approach to investment has commonly been called corporate social responsibility (CSR).

I firmly believe that a business needs a greater purpose than just making profits. CSR must be integrated into its daily thinking and activities over a sustained period. In BP's case that greater purpose became to fulfil basic human needs: to provide heat, light and mobility and to go beyond petroleum" 32 .

Même s'il s'en est défendu, le successeur de Lord Browne, Tony Hayward, a pré- féré délaisser la campagne Beyond Petroleum en insistant sur la mission première de l'entreprise : l'extraction et la distribution de pétrole, et en s'engageant plutôt à faire de la sécurité sa priorité. Bien que les documents ne soient pas de même nature, il est intéressant de mettre dos à dos les déclarations de Tony Hayward pendant la crise de Deepwater Horizon et celles de Lord Browne dans ses mémoires.

Hayward est assez loquace quant au virage sécuritaire qu'il aurait impulsé à son arrivée dans l'entreprise.

«Depuis que je suis le premier dirigeant de cette société, j'ai toujours insisté sur une exploitation sécuritaire et fiable. J'ai donné le ton au tout premier échelon de la direction en faisant clairement savoir à tout le monde chez BP que notre grande priorité était une exploitation sécuritaire et fiable [...]. Au cours des trois dernières années, nous avons investi plus de 14 milliards de dollars dans l'intégrité de nos installations. Nous nous sommes engagés dans un changement systémique chez BP durant cette période. Nous avons commencé à changer la culture [...]. Nous avons considérablement modifié les méthodes utilisées pour gérer nos activités. Et, ce qui est peut-être plus important encore, nous avons placé la sécurité et la fiabilité de l'exploitation au cœur de notre mission d'entreprise. Je le mentionne chaque fois que je prends la parole en interne et chaque fois que je parle de BP à l'extérieur de la société. Je ne nie pas qu'il y a encore place pour des améliorations, mais nous avons opéré un changement drastique au sein du personnel de notre entreprise, de ses compétences et de ses

30 J. Browne, Beyond Business: An Inspirational Memoir from a Remarkable Leader, Londres, Weidenfeld \& Nicolson, 2010.

${ }^{31}$ M. A. Cherry et J. Sneirson, "Beyond Profit", art. cit., p. 992.

32 J. Browne, Beyond Business, op. cit. 
aptitudes. Nous avons investi massivement dans ces changements $»^{33}$.

Pourtant lui-même se dégage totalement des décisions ayant mené à l'accident, comme si celles-ci n'étaient pas liées aux programmes mis en oeuvre sous sa direction, illustrant leur fonctionnement de même que leur réalité organisationnelle, audelà des discours.

« Comme je n'ai pas pris part à cette décision, je ne peux pas répondre à cette question [...]. D'après ce que j'ai lu, ce que je comprends - et encore une fois je n'ai pas été consulté pour cette décision -, c'est que [...] c'est une décision à laquelle je n'ai pas participé [...]. Je n'ai pas été impliqué dans cette décision [...]. Je n'avais pas été mis au courant ni impliqué dans les décisions pour ce puits pendant son forage [...]. Je ne sais pas qui exactement a pris la décision [...]. Je n'étais pas au courant des décisions et je n'avais pas pris connaissance des documents concernant ce puits avant ou durant le forage du puits [...]. Je n'ai participé à aucune des décisions entourant ce puits pendant le forage. Par conséquent, je ne peux pas juger si les décisions qui ont été prises étaient bonnes ou pas ».

Ce qui amène Henry Waxman, un membre de la commission (et président de la Commission de l'Énergie de la Chambre des Représentants), à conclure que Hayward semble étranger non seulement aux décisions, mais à l'entreprise elle-même :

« Je suis tout simplement renversé par ce témoignage. M. Hayward, vous n'assumez pas votre responsabilité ! Vous refusez de faire face au problème et vous agissez comme si vous n'aviez rien à voir avec cette société ou avec ses décisions ».
Or, comme l'illustre clairement le cas de $\mathrm{BP}$, les discours d'un dirigeant ne suffisent pas pour comprendre les priorités à l'œuvre dans une organisation : il est nécessaire d'enquêter sur les procédures et les mécanismes décisionnels, incitatifs et autres pour les saisir. Les travaux des multiples commissions entourant non seulement la tragédie de Deepwater Horizon mais aussi les autres accidents survenus dans la même décennie ont mis au jour la culture et le processus décisionnel de $\mathrm{BP}$ en montrant à quel point, malgré la préoccupation affichée de ses dirigeants, la sécurité devait céder le pas à l'efficacité et au contrôle des coûts au moment de prendre les décisions.

Mais l'exercice vaut également pour la fonction même de dirigeant : les résultats attendus de la part d'un PDG sont notamment lisibles à travers son système de rémunération. Et contrairement aux propos que tient Lord Browne dans ses mémoires, à l'époque de son mandat, la fonction du dirigeant de BP de même que les objectifs de l'entreprise étaient réduits de façon non équivoque à la valeur de l'action. Comme le précise le rapport annuel de 2004 de BP :

"The remuneration committee's reward policy reflects its aim to align executive directors' remuneration with shareholders' interests and to engage world-class executive talent for the benefit of the group. The main principles of the policy are: [...] Executive directors' incentives should be aligned with the interests of ordinary shareholders. This is achieved through setting performance targets that take account of measures of shareholders' interest and through the committee's policy that each executive director should hold a significant shareholding in the company, equivalent in value to $5 \mathrm{x}$ the director's base salary. The performan-

\footnotetext{
${ }^{33}$ Les citations sont la traduction d'extraits de la transcription de l'audience sur le rôle de BP dans l'explosion et le déversement de pétrole de la plate-forme Deepwater Horizon, qui s'est tenue le 17 juin 2010 devant le Subcommittee on Oversight and Investigations, Committee on Energy and Commerce, Washington, D.C. reproduits dans F. Lüdeke-Freund et D. Zvezdov, “The Manager's Job at BP”, art. cit.
} 
ce targets in the Executive Directors' Incentive Plan should encompass demanding comparisons of BP's shareholder returns and earnings with those of other companies in its own industry and in the broader marketplace" 34 .

Entièrement tourné vers les actionnaires et la rentabilité financière, le cas de BP montre avec force comment cette définition restrictive des objectifs de la firme entre fatalement en contradiction avec les intérêts des autres acteurs et de la société. La forme de rémunération prévue chez $\mathrm{BP}$, illustrative de ce qui peut être interprété comme une alliance entre les gestionnaires et les financiers $^{35}$, répond à une conception de l'entreprise qui s'est imposée à partir de la fin des années 1970, mais qui renvoie aux enjeux qu'avaient soulevés Adolf Berle et Gardiner Means en $1932^{36}$ dans leur analyse des formes modernes de la corporation $^{37}$. Il peut être utile de revenir à ce débat pour comprendre comment Lord Browne a pu tenir un discours de responsabilité sociale alors même qu'il déployait dans l'entreprise une rationalité que Tony Hayward peinait à admettre face au public américain.

En regard du bilan peu reluisant de son entreprise en matière de sécurité et d'environnement et de l'éclatement de son image verte suite à la tragédie de Deepwater Horizon, il est tentant de discréditer l'authenticité de l'engagement que Browne dépeint dans ses mémoires ${ }^{38}$. Mais un tel discrédit n'explique pas la concomitance des deux discours, c'est-à-dire les raisons pour lesquelles le discours public diffère à ce point de la rationalité interne; pourquoi cette rationalité ne peut-elle être proclamée au grand jour si elle gouverne effectivement les décisions dans l'organisation ? Ou, à l'inverse, pourquoi la rationalité interne ne correspond-elle pas aux engagements publics concernant l'environnement et la sécurité des salariés ?

Notre hypothèse est que l'ambiguïté non résolue de la nature de la firme pose le dirigeant au confluent de deux logiques contradictoires qu'il tente d'articuler : comptable face à ses actionnaires du rendement qu'il leur assure, il ne répond aux responsabilités que lui attribuent les autres acteurs sociaux qu'à travers des discours, des campagnes de relations publiques et des initiatives marginales de responsabilité sociale et de philanthropie. La « vraie » responsabilité, celle susceptible d'être sanctionnée, est bien celle vis-à-vis de l'actionnaire, et c'est elle qui structure l'organisation et se diffuse à travers la ligne hiérarchique, au détriment des impératifs de sécurité et de la protection de l'environnement.

En vue d'explorer cette hypothèse, et donc de voir comment le dirigeant conçoit l'exercice de direction, entre volonté de satisfaire ses actionnaires et désir de contribuer à des objectifs sociétaux plus larges, nous avons analysé les 21 discours officiels prononcés par Tony Hayward devant différents auditoires depuis sa nomination en mai 2007 jusqu'à la catastrophe du Golfe du Mexique en avril $2010^{39}$.

\footnotetext{
${ }^{34}$ Extrait tiré de C. Windsor et P. McNicholas, "The BP Gulf Oil Spill”, art. cit., p. 27.

${ }^{35}$ R. Boyer, Les financiers détruiront-ils le capitalisme?, Paris, Economica, 2011.

${ }^{36}$ A. Berle et G. Means, The Modern Corporation and Private Property, New York, Harcourt, Brace \& World, 1932.

${ }^{37}$ Nous utiliserons dans le reste du texte le terme de corporation dans son sens anglo-saxon plutôt que celui d'entreprise. La corporation désigne une entreprise ou un groupe d'entreprises qui est contrôlé comme une seule organisation et qui constitue une entité juridique.

38 J. Browne, Beyond Business, op. cit.

${ }^{39}$ Les discours ont été extraits du site web de l'entreprise www.bp.com le 3 juin 2013, convertis sous le format Rich Text Format, puis importés dans le logiciel d'analyse qualitative Atlas.ti qui nous a permis d'une part de dégager les thématiques principales abordées par le dirigeant et d'autre part de cerner en détail comment celles-ci sont discutées et reliées.
} 


\section{LE MÉTIER DE DIRIGEANT ET LES CHANGEMENTS CLIMATIQUES : LES DISCOURS DE TONY HAYWARD}

\subsection{Le contenu du discours du dirigeant en général}

Il ressort de notre analyse que les thématiques les plus fréquentes dans les discours de Tony Hayward sont par ordre d'importance $1 /$ les investissements, 2 /la sécurité énergétique, et $3 /$ les changements climatiques. Sans surprise, ce sont donc les investissements qui préoccupent le plus le dirigeant de BP, mais presque toujours avec en trame de fond la thématique de l'énergie, et plus particulièrement la gestion efficace des ressources énergétiques à court et à long termes dans une économie mondialisée où les États-Unis jouent un rôle prépondérant.

La sécurité énergétique apparaît ainsi comme la seconde problématique en importance dans les discours de Tony Hayward. Définie par ce dernier comme "l'accès à des sources d'énergie fiables, à un prix abordable, produites de manière responsable et respectueuse de l'environnement », la sécurité énergétique dépend de quatre impératifs : « continuer à faire fonctionner les marchés mondiaux », " développer les ressources énergétiques nationales », « investir dans les structures nationales » et « investir dans de nouvelles sources d'énergie alternatives $»^{40}$. Dans cette configuration, le dirigeant de BP considère que les principales limites au développement des réserves énergétiques nationales ne sont pas leur volume, puisqu'il estime ces dernières abondantes pour les 60 à 100 années à venir, mais plutôt les obstacles géopolitiques (ex. : la Russie et le Moyen-Orient qui possèdent $90 \%$ des réserves) et techniques (ex. : exploitation des sources non-conventionnelles et alternatives, augmentation du taux de valorisation énergétique) qui nuisent à la circulation des réserves d'énergie sur les marchés. À ce niveau, une des préoccupations majeures de Tony Hayward est le « développement de nouvelles formes de relations contractuelles qui vont au-delà du modèle historique qui requiert une appropriation des réserves et de la production ${ }^{41}$.

\subsection{Le contenu du discours du dirigeant sur les changements climatiques}

Les changements climatiques constituent la troisième thématique la plus abordée dans les discours de Tony Hayward. Celle-ci arrive aussi en tête des questions environnementales traitées par le dirigeant de $\mathrm{BP}$ en incluant les émissions de gaz à effet de serre, qui sont identifiées comme la cause essentielle du réchauffement climatique. À ce sujet, le dirigeant déclare être tout à fait conscient que l'industrie pétrolière est responsable de $60 \%$ des émissions de gaz à effet de serre, mais il rappelle que BP, rebaptisée Beyond Petroleum, entreprendra les actions nécessaires pour atteindre une économie à faible émission de carbone. Pour Tony Hayward en effet, agir pour mitiger les changements climatiques devient urgent en regard de la demande énergétique mondiale croissante susceptible de les amplifier ${ }^{42}$.

Pour ce faire, Hayward souligne la nécessité d'une implication gouvernementale. Il en appelle tout d'abord à ce que les gouvernements mettent davantage l'accent sur des mécanismes de marché susceptibles,

\footnotetext{
${ }^{40}$ T. Hayward, "Energy Security and America", The Houston Forum, Houston, USA, 8 November 2007.

41 T. Hayward, World Petroleum Council, Madrid, Spain, 30 June 2008.

42 T. Hayward, "Energy security through diversity", Prepared remarks delivered at a Peterson Institute event, Peterson Institute, USA, 23 March 2010.
} 
par la concurrence, de créer un prix pour le carbone et donc de limiter les émissions de gaz à effet de serre ${ }^{43}$. Mais il croit également que les législateurs devraient déployer un cadre régulatoire clair et stable, capable de susciter l'investissement - que ce soit dans les énergies à faible empreinte carbone ou dans les énergies fossiles.

C'est souvent en ce sens qu'il aborde le potentiel des énergies renouvelables et alternatives, thématique présente dans 14 des 21 discours analysés. Tony Hayward parle en effet très souvent des biocarburants, et de différents types d'énergies renouvelables, comme de pistes d'avenir, mais explique à quelques reprises que plusieurs d'entre elles demeurent encore trop coûteuses pour être exploitées plus largement et qu'il faudrait, pour limiter les changements climatiques, privilégier les énergies les plus abordables telles que le nucléaire, le gaz naturel ou encore l'énergie éolienne onshore ${ }^{44}$.

Il en va de même pour la problématique de la capture et du stockage du carbone (CSC) qui est souvent évoquée. À l'identique des énergies renouvelables, la technologie CSC est tantôt présentée comme une alternative très importante, tantôt comme une technologie qui ne verra pas le jour avant 2030 voire plus tard, tantôt comme une technologie encore trop coûteuse.

"In my view it's vital, as we gear up to confront the problem of climate change, that we choose the lowest-cost energy pathways. Energy efficiency, gas fired power, lighter cars and advanced biofuels all offer relatively low-cost routes, while more headline-grabbing options are not the most cost-effective in terms of cost per ton of mitigated $\mathrm{CO}_{2}$. With today's technology, carbon capture and storage to make clean coal, for example, is very expensive. Offshore wind is also costly - for example in comparison to onshore wind, which is now a big business for BP in the United States, and indeed to nuclear".

\subsection{Le contenu du discours du dirigeant sur les questions relatives à la sécurité}

Ce qui peut paraître surprenant est le fait que les questions de « sécurité » font partie des thèmes les moins abordés dans les discours de Tony Hayward, alors qu'il disait en faire sa priorité. Si bien que malgré son affirmation selon laquelle la sécurité devrait être «la priorité $\mathrm{n}^{\circ} 1$ », ses discours traitent rarement de la sécurité au travail. Peu après son arrivée au poste de CEO, Tony Hayward reconnaît pourtant que, durant les années précédentes, BP a connu des failles importantes de sécurité. En tirant des leçons de l'accident tragique de la raffinerie de Texas City de mars 2005, des suites duquel 15 ouviers avaient péri et des centaines d'autres avaient été blessés, il considère que cet accident a marqué un tournant essentiel dans l'histoire de BP. Il qualifie ces failles de sécurité à la fois de "manquements graves » et d' « énorme opportunité d'apprendre et d'améliorer la façon dont BP exerce ses activités » et il s'engage à continuer de mettre en œuvre la feuille de route établie par le Baker Panel Report ${ }^{45}$.

\subsection{Les discours du dirigeant confrontés aux faits}

De par sa vocation politique et idéologique, un discours peut être en décalage avec la réalité de l'organisation. Néanmoins il permet de dégager un certain nombre de comportements et d'intentions des diri-

\footnotetext{
${ }^{43}$ Idem.

44 T. Hayward, "The Challenge of Energy Security”, London Business School, London, UK, 4 February 2010.

45 T. Hayward, "Energy Security and America”, The Houston Forum, Houston, USA, 8 November 2007.
} 
geants. Dans le cas des discours de Tony Hayward, nous avons pu remarquer deux aspects importants : d'une part une certaine incohérence dans le contenu des discours et d'autre part une orientation des propos tenus en fonction de l'audience.

L'incohérence, imperceptible si on analyse chaque discours de façon isolée, ressort d'une analyse d'ensemble, ou même de comparaisons plus ponctuelles. Selon l'auditoire et la chronologie des discours, les propos se contredisent, tout spécialement à propos des questions environnementales et sociales. À titre d'exemple, Hayward déclare en 2008 : «La sécurité est notre priorité $n^{\circ} 1 »^{46}$. C'est également ce qu'il affirme un an plus tard en expliquant ${ }^{47}:$ " Notre priorité $n^{\circ} 1$ pour une exploitation sûre et fiable a été vitale pour le développement de notre compétitivité ». Cependant, dix mois plus $\operatorname{tard}^{48}$, la priorité $n^{\circ} 1$ selon Hayward n'est plus la sécurité mais l'efficience énergétique. Il établit en effet que « l'efficacité énergétique est la priorité $n^{\circ} 1 »$. Mais peu de temps après ${ }^{49}$, la sécurité redevient la priorité $n^{\circ} 1$. Il affirme en effet que « [...] fournir un environnement de travail sécurisé est une responsabilité suprême, et constitue notre priorité absolue $»^{50}$. On peut aussi noter les copier-coller d'un discours à l'autre qui concernent essentiellement les questions environnementales et sociales de l'entreprise, ce qui laisse penser qu'il s'agit de la portion la moins maîtrisée par le dirigeant qui reprend telles quelles les notes qu'on lui a préparées. Ainsi, l'expression "Carbon pricing will make energy conservation more attractive and alternative energy more cost competitive. It will allow informed investment in fossil fuels and will encourage investment in the technology necessary to reduce the carbon they produce" a été utilisée à l'identique lors de trois discours ${ }^{51}$.

Les contradictions dans le discours sont aussi très évidentes quand le dirigeant parle des énergies renouvelables. D'un côté, Tony Hayward affirme : « la part des énergies renouvelables augmentera certainement $»^{52}$, « les énergies renouvelables vont jouer un rôle important » et « l'énergie renouvelable constituera une part importante du futur bouquet énergétique ${ }^{53}$. Il déclare même que «les énergies renouvelables comme l'énergie éolienne et l'énergie solaire vont jouer un rôle de plus en plus important $»^{54}$ ou encore : "Nous ne pouvons pas nous permettre d'attendre. Nous devons commencer à enlever le carbone du mix énergétique aujourd'hui » ${ }^{55}$. D'un autre côté, le dirigeant énonce des propos inverses : « les technologies, les infrastructures et le cadre réglementaire de ces énergies alternatives devraient prendre des décennies à être

\footnotetext{
46 T. Hayward, "Energy Security and America". The Houston Forum, Houston, USA, 8 November 2007.

${ }^{47}$ T. Hayward, Speech at the 2008 Annual General Meeting, ExCel centre, Docklands, London, UK, 17 April 2008.

${ }^{48}$ T. Hayward, Speech at the 2009 Annual General Meeting, ExCel centre, Docklands, London, UK, 16 April 2009.

49 T. Hayward, "The Challenge of Energy Security", London Business School, London, UK, 4 February 2010.

50 T. Hayward, "Energy security through diversity", Prepared remarks delivered at a Peterson Institute event, Peterson Institute, USA, 23 March 2010.

51 T. Hayward, "Meeting the Energy Challenge", Oil and Money Conference, London, UK, 20 October 2009; T. Hayward, "The harsh realities of energy", MIT, Boston, USA, 29 October 2009; T. Hayward, "Energy pathways Setting a course to a sustainable energy future”, World Oil and Gas Assembly, Bangalore, India, 4 December 2009.

52 T. Hayward, "The Role of Gas in the Future of Energy", World Gas Conference, Buenos Aires, Argentina, 08 October 2009; T. Hayward, "Energy security through diversity", Prepared remarks delivered at a Peterson Institute event, Peterson Institute, USA, 23 March 2010.

53 T. Hayward, "The harsh realities of energy”, MIT, Boston, USA, 29 October 2009.

54 T. Hayward, "Meeting the Energy Challenge", Oil and Money Conference, London, UK, 20 October 2009.

55 Idem.
} 
déployés à grande échelle $»^{56}$ ou bien : «il est difficile de penser que les énergies renouvelables vont compter pour plus de $5 \%$ de la consommation énergétique en $2030 »^{57}$ ou encore : « toutes ces technologies sont de temps en temps promues d'une manière qui suggérerait qu'elles sont l'avenir. Mais il n'y a pas de solution miracle $»^{58}$ et enfin : " il y a des limites réelles de la capacité physique, de l'ingénierie et de l'économie à surmonter ${ }^{59}$.

Par ailleurs, on peut se demander pourquoi les propos sur les énergies renouvelables sont omniprésents dans les discours de Tony Hayward, malgré des investissements réels minimes dans ces énergies de la part de l'entreprise. On peut faire l'hypothèse qu'il s'agit surtout de rhétorique, comme le laissent entrevoir les propos du dirigeant lui-même : "we need to demonstrate that we are part of the solution, not the problem" 60 .

Très souvent les messages de Tony Hayward sur la technologie CSC sont aussi contradictoires. Par exemple : " [et la capture et le stockage du carbone entre autres] non seulement vont aider à s'attaquer aux changements climatiques mais contribueront également à diversifier et à accroître l'offre d'énergie ${ }^{61}$, alors que l'année suivante il déclare : "Bien que la capture et le stockage du carbone soient souvent présentés comme une voie menant vers la combustion propre du charbon, ils restent confrontés à un certain nombre de défis qui nécessiteront du temps et des efforts pour être résolus ${ }^{62}$. Lors de trois de ses discours $^{63}$, il déclare : « en 2030, nous prévoyons que la technologie de capture du carbone pourrait être déployée à grande échelle».

Mais, outre les incohérences tout juste présentées, force est de constater que certaines thématiques sont abordées plus que d'autres devant certains types d'auditoire. Ainsi, il ressort de notre analyse que les questions environnementales et sociales de l'entreprise sont évoquées plus souvent lors des discours prononcés à l'occasion de conférences internationales que lors des rencontres avec des étudiants et des chercheurs, et encore moins lors des assemblées générales. On observe par exemple que $37 \%$ des propos tenus sur les énergies renouvelables et alternatives l'ont été lors de conférences internationales, un peu moins lors de discours devant des étudiants et des chercheurs $(30,3$ et $22,4 \%$ respectivement), alors que la part de ces mêmes propos lors des discours devant les actionnaires n'est que $10,1 \%$. Des proportions semblables valent pour les questions environnementales, l'engagement communautaire et l'énergie à faible teneur en carbone. Ce sont les thématiques sur le «profit» et la « sécurité » qui font la différence, alors que Tony Hayward n'en parle que devant ses actionnaires ( $75 \%$ et $71,4 \%$ de la part des propos respectivement). On peut aussi

\footnotetext{
56 T. Hayward, "The harsh realities of energy”, MIT, Boston, USA, 29 October 2009.

${ }^{57}$ T. Hayward, "Energy pathways-Setting a course to a sustainable energy future", World Oil and Gas Assembly, Bangalore, India, 4 December 2009.

58 T. Hayward, "The harsh realities of energy", MIT, Boston, USA, 29 October 2009.

59 Ibid.

60 T. Hayward, "Energy Security and America", The Houston Forum, Houston, USA, 8 November 2007.

61 T. Hayward, "How to expand energy supply in the 21st century", 13th Asia Oil \& Gas Conference, Kuala Lumpur, Malaysia, 9 June 2008.

62 T. Hayward, "The Role of Gas in the Future of Energy", World Gas Conference, Buenos Aires, Argentina, 8 October 2009.

63 T. Hayward, "Energy pathways-Setting a course to a sustainable energy future", World Oil and Gas Assembly, Bangalore, India, 4 December 2009; T. Hayward, "The Challenge of Energy Security", London Business School, London, UK, 4 February 2010; T. Hayward, "Energy security through diversity", Prepared remarks delivered at a Peterson Institute event, Peterson Institute, USA, 23 March 2010.
} 
observer que lors de discours devant des étudiants, la part des propos sur les énergies renouvelables, comparée à d'autres types de propos, est de 50,8 \%, suivie des questions environnementales $(21,8 \%)$ et de l'engagement communautaire $(12,1 \%)$; le dirigeant n'y mentionne pas du tout ni la thématique du profit ni celle de la sécurité.

On peut remarquer enfin que les discours de Tony Hayward sont extrêmement précis lorsqu'il évoque des chiffres liés à son métier de production d'énergie à partir des sources fossiles, tandis que l'imprécision est frappante lorsqu'il évoque les questions environnementales et les énergies renouvelables.

Cette analyse montre combien la responsabilité du dirigeant s'ancre d'abord dans le tandem du cœur de métier et des résultats financiers, au détriment de la sécurité, de la protection de l'environnement, de la transition énergétique et du grand enjeu de société que sont les changements climatiques. Bien qu'elle soit présentée comme un enjeu objectif auquel il convient de répondre au plus vite, la lutte contre le réchauffement climatique ne se matérialise ainsi en actions concrètes que si elle n'a pas d'incidence sur le rendement financier. Le dirigeant ne semble donc pas prêt à mettre en place toutes les actions nécessaires à l'avènement d'une faible économie de carbone.

\section{ENTREPRISE PRIVÉE OU INSTITUTION PUBLIQUE : LE DIRIGEANT AU CFUR DU PARADOXE DE LA CORPORATION MODERNE}

Cette représentation ambiguë du rôle de l'entreprise face à un enjeu dont elle est pourtant en grande partie responsable est inspirée par une vision de la firme dominante depuis vingt ans : la firme comme nœud de contrats ${ }^{64}$. Un regard historique permet en effet de voir que les acteurs et donc la nature de l'entreprise ont considérablement évolué depuis le début du siècle dernier. L'entreprise n'est plus une petite entité familiale dirigée par une seule et même personne ou par quelques personnes en interaction constante au même endroit dans un contexte de fort encadrement étatique. Depuis l'avènement de la grande entreprise, l'entreprise est dirigée par le gestionnaire mais financée par les marchés financiers, où le gestionnaire serait un simple agent et l'actionnaire l'acteur dont l'intérêt - financier - apparaît comme le seul objectif légitime de l'entreprise ${ }^{65}$. Cette vision de l'entreprise, relayée notamment par les dispositifs légaux et véhiculée dans la sphère publique, de même que dans les écoles de gestion, engage et légitime le dirigeant et son activité dans un cadre balisé par des indicateurs financiers, alors que ses décisions sont motivées, évaluées et justifiées par cet objectif de premier ordre ${ }^{66}$. La représentation dominante de l'entreprise construit ainsi une rationalisation paradoxale de son processus décisionnel : la mission de l'entreprise traduit un équilibre des intérêts, mais un seul acteur a un intérêt légiti-

\footnotetext{
${ }^{64} \mathrm{~V}$. Chassagnon, «Une analyse historique de la nature juridique de la firme », Revue de la régulation, vol. 12, $2^{\mathrm{e}}$ semestre / Autumn 2012. En ligne : http:// regulation.revues.org/9832. Voir aussi B. Segrestin, B. Roger et S. Vernac (dir.), L'entreprise : point aveugle du savoir, Auxerre, Éditions Sciences Humaines, 2014.

65 W. Bratton et M. L. Wachter, "Shareholder Primacy's Corporatist Origins: Adolf Berle and 'The Modern Corporation"”, Journal of Corporation Law, vol. 34, n 1, 2008, p. 99-152.

${ }^{66}$ M. C. Jensen, "Value Maximisation, Stakeholder Theory, and the Corporate Objective Function", Business Ethics Quarterly, vol. 12, n², 2002, p. 235-256.
} 
me à faire valoir au sein de la firme : l'actionnaire ${ }^{67}$. C'est une représentation qui interdit de penser d'une part les questionnements sur sa gouvernance, en déterminant, une fois pour toutes, le seul acteur ayant droit de regard sur la firme, mais aussi d'autre part les questionnements sur son projet, incarnation d'un équilibre qu'on ne saurait profaner.

Souvent présentée comme objective, cette définition de l'entreprise est pourtant critiquée par un nombre croissant d'acteurs et de chercheurs, pour ses implications performatives d'une part ${ }^{68}$, mais aussi pour son inexactitude juridique. En effet, plusieurs ont rappelé qu'en regard du droit, l'actionnaire n'est pas plus propriétaire de l'entreprise que le dirigeant n'est son agent ${ }^{69}$. Certains tribunaux ont aussi précisé que l'intérêt de la firme ne peut se résumer à celui de ses actionnaires ${ }^{70}$. Simultanément, l'entreprise fait l'objet depuis ces dernières années de multiples requêtes à travers lesquelles on l'enjoint de tenir compte de ses parties prenantes et d'élargir la mesure de sa performance à de nouvelles dimensions sociale et environnementale.

$\mathrm{Si}$ bien que les gestionnaires doivent aujourd'hui faire face à des injonctions contradictoires, comme l'illustre bien la Commission où fut entendu Hayward, injonctions contradictoires que tente notamment de résoudre le courant Business Case de la responsabilité sociale en arguant qu' « il est rentable d'être responsable ». Cette dissonance semble pourtant à l'origine d'un nouveau malaise gestionnaire qu'ont récemment dépeint plusieurs auteurs issus de courants les plus divers ${ }^{71}$ et que l'on peut qualifier de souffrance téléologique ${ }^{72}$. Les gestionnaires doivent en effet s'inscrire dans les paramètres que dicte la représentation dominante, mais leur travail quotidien les confronte à une réalité qui l'éclate sans qu'ils puissent s'appuyer sur une autre représentation cohérente de la firme.

Critiques de la théorie dominante, les chercheurs en responsabilité sociale ont proposé des perspectives alternatives de l'entreprise susceptibles de refonder les responsabilités du dirigeant sur un spectre plus large que les seuls indicateurs financiers. Fragmentées et contradictoires, ces conceptions n'ont néanmoins pas encore réussi à se cristalliser en une définition alternative de l'entreprise.

Et même l'approche des parties prenantes ${ }^{73}$, qui est probablement la plus diffu-

\footnotetext{
${ }^{67}$ C. Gendron, « Grande entreprise et gouvernement des sociétés. De l'acteur économique à l'institution sociale », in J.-P. Chanteau, K. Martin-Chenut et M. Capron (dir.), Entreprise et responsabilité sociale en question. Savoirs et controverses, Paris, Classiques Garnier, 2017, p. 285-300.

${ }^{68}$ M. Callon, The Laws of the Markets, Oxford, Blackwell Publishers, 1988.

${ }^{69}$ Notamment L. A. Stout, "Bad and Not-So-Bad Arguments For Shareholder Primacy", Southern California Law Review, vol. 75, 2002, p. 1189-2002 ; mais aussi C. Gendron, « Vers une approche politique de l'entreprise », in B. Segrestin et alii (dir.), L'entreprise, op. cit.

${ }^{70}$ I. B. Lee, "Peoples Department Stores V. Wise and the 'Best Interests of the Corporation", Canadian Business Law Journal, vol. 41, n² 212, 2005; S. Rousseau et I. Tchotourian, « Pouvoirs, institution et gouvernance de la société par actions : lorsque le Canada remet en question le dogme de la primauté actionnariale ", Les cahiers de la $C R S D D$, vol. 5, $\mathrm{n}^{\circ} 34,2012$; et $\mathrm{S}$. Rousseau et I. Tchotourian, «'L'intérêt social' en droit des sociétés : regards transatlantiques », Revue des sociétés, vol. 4, 2009, p. 735-760.

${ }^{71}$ Notamment F. Dupuy, La fatigue des élites, Paris, Éditions du Seuil, 2005; C. Dejours, Souffrance en France. La banalisation de l'injustice sociale, Paris, Éditions du Seuil, 1998, p. 183; C. Cederström et P. Fleming, « Dead Man Working »,M@n@gement, vol. 15,n², 2012,p.452-458.

72 C. Gendron, « La norme ISO 26000, une avancée dans la prise en compte de la santé au travail. Peut-on penser la souffrance au travail à la lumière de la responsabilité sociale ? », in P. Bardelli et J. Allouche (dir.), La souffrance au travail : quelle responsabilité de l'entreprise?, Paris, Armand Colin, 2012, p. 31-55.

${ }^{73}$ R. E. Freeman, Strategic management: A stakeholder approach, Boston, Pitman, 1984.
} 
sée, tend à être interprétée dans des termes contractuels qui la rapprochent de la théorie dominante ${ }^{74}$.

Une avenue reste encore peu explorée, même si elle s'appuie sur des textes parfois abondamment cités : celle de l'entreprise comme institution sociale. Paradoxalement, c'est aux origines de la théorie de l'agence qu'on peut trouver cette idée d'une entreprise comme institution. En effet, dès 1932, Adolf Berle et Gardiner Means ${ }^{75}$, souvent cités comme étant à l'origine de la théorie de l'agence ${ }^{76}$, décrivent cette nouvelle forme qu'est l'entreprise moderne.

L'entreprise ne se résume plus ici à une activité économique, c'est une institution publique qui doit embrasser une finalité plurielle répondant à des intérêts commerciaux définis de manière large. E. Merrick Dodd en $1941^{77}$ poursuit la réflexion en définissant l'entreprise comme une institution économique combinant une activité sociale et une fonction lucrative, ce qu'Alain Touraine formalisera trente ans plus tard de la manière suivante : «Institution privée remplissant une fonction sociale ; intention de rationalité gérée par un système politique privé ${ }^{78}$.

Cette définition fait écho à la dualité trop souvent niée de la généalogie de l'entreprise, tout autant fille de la corporation constituée par des privilèges accordés par l'église médiévale et les États souverains que des regroupements professionnels ou artisans permis par la liberté d'association.
Cette origine duale peut expliquer ce que l'on peut décrire comme «le conflit actuel sur la nature, les pouvoirs et les responsabilités des entreprises ${ }^{79}$. Envisager l'entreprise comme institution sociale permet de saisir qu'elle est au cœur de conflits et de rapports de pouvoir, rapports qu'elle structure et pour lesquels elle propose un compromis temporaire. D'où sa nature perpétuellement controversée.

L'entreprise est donc l'expression d'une hégémonie qui profite à cette nouvelle alliance entre les gestionnaires et les dirigeants ${ }^{80}$, que confirment le rapport entre la hausse des rémunérations des gestionnaires ainsi que la profitabilité des marchés financiers (incluant la rémunération de ses opérateurs) d'une part et l'élimination des emplois ou la réduction des salaires dans la foulée de la mondialisation et de sa rhétorique de compétitivité d'autre part.

Mais l'hégémonie suppose aussi une légitimation que peine à maintenir le système actuel, malgré ses efforts de communications. L'entreprise envisagée comme institution sociale doit s'inscrire dans un projet collectif, lequel ne peut plus être réduit à la mécanique vertueuse du marché. Dans ce cadre, les gestionnaires sont propulsés au rang de « dirigeants sociaux » ${ }^{81}$ et appréhendés comme tels par le public ou dans le cadre de commissions telles que la National Commission on the BP Deepwater Horizon Oil Spill and Offshore Drilling.

\footnotetext{
${ }^{74}$ A. Acquier, « Théorie des parties prenantes et RSE : quel agenda pour l'École de Montréal ? », in C. Gendron et B. Girard (dir.), Repenser la responsabilité sociale avec l'École de Montréal, Paris, Armand Colin, 2013.

75 A. Berle et G. Means, The Modern Corporation and Private Property, op. cit., p. 309-310.

${ }^{76}$ Filiation que nous jugeons usurpée, à l'instar de W. Bratton et W. Wachter, "Shareholder Primacy's Corporatist Origins", art. cit.

${ }^{77}$ E. M. Dodd, "The modern corporation, private property, and recent federal legislation", Harvard Law Review, vol. 54, nº 6, 1941, p. 917-948.

${ }^{78}$ A. Touraine, La société post-industrielle, Paris, Denoël, 1969.

${ }^{79}$ R. Eels et C. C. Walton, Conceptual Foundations of Business, Homewood (Ill.), Richard D. Irwin, 1961.

${ }^{80} \mathrm{~T}$. Bower, The squeeze: oil money and greed in the 21 st century, Londres, Harper Press, 2009.

${ }^{81}$ A. Berle et G. Means, The Modern Corporation and Private Property, op. cit. ; A. Touraine, La société postindustrielle, op. cit. ; A. Sales, « L'entreprise et son environnement ». Sociologie et Sociétés, vol. 2, n 1, mai 1970, p. 107-121.
} 
Or le droit ne rend pas bien compte de ce rôle élargi du dirigeant, car il tend à mettre l'accent sur un lien quasi-fiduciaire avec les actionnaires $^{82}$. Il est néanmoins pertinent de suivre certaines évolutions récentes qui pourraient transformer la responsabilité du dirigeant en y inscrivant, de manière directe, des objectifs vis-à-vis de l'environnement et des autres acteurs sociaux. À titre d'illustration, les développements judiciaires canadiens (les arrêts BCE et People) statuent que la responsabilité du dirigeant s'exerce à l'égard non seulement des actionnaires, mais encore de l'ensemble des parties prenantes, et qu'ils n'ont donc pas la préséance dans la définition de l'intérêt de l'entreprise ${ }^{83}$. D'autres initiatives tendent à inscrire les résultats extra-financiers au chapitre des résultats sanctionnables de l'entreprise $^{84}$. La question sera alors de savoir comment les différents objectifs de l'entreprise devront être pondérés, et s'il sera même possible de les considérer simultanément pour orienter efficacement l'organisation $^{85}$.

Si l'arbitrage ne peut se faire à l'échelle organisationnelle, il devra vraisemblablement être stabilisé à travers des règles institutionnelles, c'est-à-dire au cœur même de la définition de l'entreprise qu'instaure le droit corporatif. Dans ce nouvel ordre corporatif, la valeur pour l'actionnaire ne serait plus un objectif de premier ordre, et, loin d'une logique privée de fiduciaire, le dirigeant pourrait être formellement réhabilité au rang de dirigeant social. De multiples questions pourraient alors se poser quant à la formation requise, et au mode de nomination à cette fonction. Quant à la faisabilité de cette évolution, elle sera intrinsèquement liée à un marché financier dont l'emprise participe de la configuration fiduciaire actuelle. Si bien que ce n'est qu'à travers sa transformation, que ce soit dans l'ajustement de ses temporalités ou l'intégration de critères extra-financiers, que le rôle du dirigeant pourra évoluer.

Miser sur la participation de l'entreprise à un marché de carbone pour atténuer le changement climatique - la solution pour de nombreux dirigeants incluant, comme nous le précisions plus tôt, Tony Hayward - ne constituerait en fait qu'un remède inefficace au problème de fond que constitue le rôle de fiduciaire du dirigeant d'entreprise. En effet, alors que c'est la configuration actuelle même du marché financier qui brime l'élan social du dirigeant, pourquoi croire, en vue de raviver cet élan, en un marché parallèle articulé selon la même logique économique qui donne lieu aux mêmes incohérences et qui minimise finalement l'urgence du problème posé par le changement climatique ${ }^{86}$ ? Dans le même ordre d'idées, si rendre le dirigeant plus soucieux des causes sociales et environnementales qui l'entourent passe par la transformation du marché financier, quel rôle viendrait y jouer un marché de carbone, dont le pilotage s'avère déjà compliqué dans le contexte de coopération européen et qu'il serait donc périlleux d'envisager à l'échelle internationale ${ }^{87}$ ?

Ce serait pourtant faire preuve de bon sens que d'amorcer au plus vite un virage pour transiter vers une économie bas carbone. En effet, les sommes à investir aujour-

\footnotetext{
${ }^{82}$ M. A. Cherry et J. Sneirson, "Beyond Profit", art. cit.

${ }^{83}$ S. Rousseau et I. Tchotourian, «'L'intérêt social' en droit des sociétés », art. cit..

${ }^{84}$ M. A. Cherry et J. Sneirson, "Beyond Profit", art. cit.

${ }^{85}$ M. C. Jensen est convaincu du contraire : « il est logiquement impossible de maximiser dans plus d'une dimension à la fois, à l'exception du cas où ces dimensions sont des transformations monotones l'une de l'autre ». M. C. Jensen, "Value Maximisation", art. cit.

${ }^{86}$ A. Pottier, Comment les économistes réchauffent la planète, Paris, Éditions du Seuil, 2016.

87 Ibid.
} 
d'hui pour limiter le changement climatique en décarbonant l'économie sont du même ordre que celles qu'il va falloir mobiliser de toute façon pour poursuivre la trajectoire actuelle. Ainsi, rester sous la barre des $2{ }^{\circ} \mathrm{C}$ coûterait 93000 milliards de dollars d'investissements sur 15 ans, contre 89000 milliards pour une trajectoire inchangée ${ }^{88}$. Mais ces sommes s'avèrent même minimes en comparaison de celles qu'il faudra défrayer dans quelques années pour pallier les impacts du changement climatique si nous ne faisons rien. D'autant plus que la question n'est pas de trouver des fonds là où il n'y en a pas puisqu'il existe, comme le font remarquer Alain Grandjean et Mireille Martini ${ }^{89}$, une épargne importante du côté des ménages dans les pays développés et émergents, et une capacité de financement colossale chez les entreprises qui préfèrent utiliser ces fonds pour racheter leurs propres actions plutôt que de procéder à de nouveaux investissements.

Le problème actuel tient en fait à deux éléments. D'une part, l'endettement passé des États amène ces derniers à limiter de nouveaux investissements, même dans des technologies vertes pourtant susceptibles de se traduire en économies d'énergie et de limiter les émissions de GES ${ }^{90}$. D'autre part, le manque d'intérêt chez les consommateurs et les entreprises afin de diriger leurs investissements vers une économie bas carbone qui offre des rendements moins élevés que ce que propose l'économie carbone actuelle reste patent ${ }^{91}$.

Sous cet angle, la solution passerait alors par une réorientation des flux finan- ciers qui sont destinés aux industries et services qui mobilisent des énergies fossiles vers des initiatives de recherche et de développement qui visent l'efficience énergétique dans les domaines des transports ${ }^{92}$ et de l'habitation pour limiter les émissions de GES $^{92}$. Pour A. Grandjean et M. Martini, cette réorientation nécessite une réglementation plus stricte des marchés financiers par les États, une réforme du système monétaire international pour envisager par exemple de ne pas comptabiliser des investissements verts dans le calcul du déficit public des États, et une implication forte, assortie d'une volonté de lutter contre le réchauffement climatique, des entreprises et de l'ensemble de la société, notamment en matière d'investissement responsable.

C'est sur ce dernier point qu'insiste le média Novethic ${ }^{93}$ en montrant que la transition vers une économie bas carbone ne passe plus seulement par une réorientation des flux financiers vers des efforts d'efficience : la réorientation de ces flux financiers peut aussi s'effectuer vers des industries et services qui tablent moins ou plus du tout sur les combustibles fossiles. Ainsi, pour les encourager ou au contraire les boycotter, un nombre croissant d'investisseurs institutionnels impliqués dans la lutte contre le changement climatique ont recours à diverses stratégies, parfois menées conjointement : certains cherchent à décarboner leurs portefeuilles en retirant les placements qu'ils avaient effectués dans les entreprises les plus polluantes, en signalant à ces dernières leur mécontentement et leur intention de désinvestir si aucune mesure n'est prise

\footnotetext{
${ }^{88}$ A. Grandjean et M. Martini, Financer la transition énergétique, Ivry-sur-Seine, Éditions de 1'Atelier, 2016.

89 Ibid.

90 Ibid.

91 Ibid.

92 A. Grandjean et M. Martini, Financer la transition, op . cit.

93 Pour en savoir davantage sur l'investissement institutionnel et le climat : http://www.novethic.fr/climat/financeet-climat.html. Pour en savoir davantage sur les acteurs les plus engagés et les chiffres de la finance liée au climat : http://www.novethic.fr/fileadmin/user_upload/tx_ausynovethicetudes/pdf_syntheses/novethic-expert-finance-etclimat.pdf.
} 
pour y répondre, en s'associant à d'autres actionnaires pour que leurs revendications aient ensemble plus de poids, ou encore en préférant investir dans des entreprises et services qui luttent contre le réchauffement climatique ou se passent carrément de combustibles fossiles.

C'est donc en valorisant davantage chez l'actionnaire les causes sociales et environnementales telles que la lutte contre les changements climatiques, et en lui permettant de bénéficier d'un rendement intéressant, que le marché sera le plus à même de faire croître un intérêt pour ces causes chez le dirigeant d'entreprise pour réhabiliter celle-ci, en tant qu'institution sociale. Reste donc à déterminer les moyens à mettre en place, en concomitance avec une réforme du Fonds monétaire international, des incita- tions à l'investissement vert par les États et une meilleure réglementation des marchés, pour susciter un tel engouement chez les entreprises et les ménages, et faire de ces derniers des actionnaires plus engagés.

Les banques ont un rôle essentiel à jouer dans cette aventure, ce que plusieurs d'entre elles, accompagnées de grands assureurs et de fonds de pension, assument déjà depuis quelque temps en se désengageant du charbon ${ }^{94}$. Mais c'est également dans le développement de financements verts attractifs comme les green bonds, dans la généralisation de nouvelles obligations de reporting sur l'intégration de critères environnementaux, sociaux et de gouvernance $(\mathrm{ESG})^{95}$ et bien sûr dans la formation des futurs dirigeants que des solutions semblent s'esquisser.

\footnotetext{
94 Novethic. 2016. « Novethic décode les liens entre finance et climat » : http://www.novethic.fr/fileadmin/user_ upload/tx_ausynovethicetudes/pdf_syntheses/novethic-expert-finance-et-climat.pdf

95 Tel que dans l'article 173 de la loi française sur la transition énergétique. Cette loi du 17 août 2015 définit dans son article 173 les obligations d'information de plus de 840 investisseurs institutionnels sur leur prise en compte de critères environnementaux, sociaux et de gouvernance (ESG). Voir à ce sujet le site de Novethic : http://www.novethic.fr/lexique/detail/article-173-1.html
} 